Revue d'histoire de l'enfance « irrégulière »

Le Temps de l'histoire

22 | 2020

Enfances (dé)colonisées

\title{
75 ans de pédopsychiatrie à Lausanne. Du Bercail au Centre psychothérapeutique
}

\section{Martine Ruchat}

\section{(2) OpenEdition}

Édition électronique

URL : https://journals.openedition.org/rhei/5416

DOI : $10.4000 /$ rhei.5416

ISSN : $1777-540 \mathrm{X}$

Éditeur

Presses universitaires de Rennes

\section{Édition imprimée}

Date de publication : 25 novembre 2020

Pagination : 173-176

ISBN : 978-2-7535-8108-1

ISSN : $1287-2431$

Référence électronique

Martine Ruchat, « 75 ans de pédopsychiatrie à Lausanne. Du Bercail au Centre psychothérapeutique », Revue d'histoire de l'enfance « irrégulière » [En ligne], 22 | 2020, mis en ligne le 25 novembre 2020 , consulté le 07 décembre 2022. URL : http://journals.openedition.org/rhei/5416 ; DOI : https://doi.org/ $10.4000 /$ rhei.5416 


\title{
Comptes rendus d'ouvrages
}

\author{
75 ans de pédopsychiatrie à Lausanne. \\ Du Bercail au Centre psychothérapeutique \\ Taline Garibian \\ 2015
}

Éditions BHMS

Dans l'histoire de l'enfance, le temps est à la commémoration car nombre d'institutions et d'établissements éducatifs et de soins furent créés il y a 75 ans, et même plus. La pédagogue suédoise Ellen Kay qualifiait le $\mathrm{xx}^{\mathrm{e}}$ siècle de « siècle de l'enfant "; je l'ai personnellement toujours considéré comme étant bien plutôt celui des professionnel.le.s de l'enfance. L'ouvrage de Taline Garibian le confirme. Car cette histoire du Bercail, première institution psychiatrique pour enfants, et de l'Office médico-pédagogie vaudois en Suisse romande, racontée de 1938 à 2013, met en jeu nombre d'acteurs et d'actrices : les enseignants spécialisés, les éducateurs, les assistantes sociales, les médecins et les psychologues. Puis viendront les logopédistes, les orthophonistes et les psychothérapeutes. Comme l'autrice l'écrit d'entrée, ce sont bien elles et eux qui écrivent l'histoire des enfants.

Cette histoire n'est d'ailleurs pas facile à écrire, tant elle se compose de champs professionnels et épistémologiques divers. Se mêlent une histoire des idées, dont le Pr François Ansermet rappelle en préface les grandes lignes dans le domaine de la pédopsychiatrie vaudoise, et une histoire des pratiques thérapeutiques - dessins, psychodrames, thérapies familiales, tests, scoutisme - qui, toutes deux, alimentent une histoire de la clinique psychiatrique infanto-juvénile et de ses catégories nosographiques. C'est aussi une histoire politique, qui structure le champ et le fait exister à travers les budgets et les règlements (notons que les institutions liées à l'enfance sont sous le Département de justice et police avant de passer sous celui de l'intérieur).

L'ouvrage de Taline Garibian est un ouvrage commémoratif, relevant donc d'un genre particulier, sorte de chronique institutionnelle s'appuyant sur des archives (administratives et médicales essentiellement) et des témoignages 
1. Voir Boussion S., Coffin J. C., "Le psychiatre, l'enfant et l'État », Revue d'histoire de l'enfance «irrégulière », Rennes, PUR, $n^{\circ} 18,2016$. oraux. Il propose d'abord une description fine des événements, sans jugement, sans prendre position. Ce livre est une pierre importante dans l'histoire de la construction du champ en Suisse, qui permet de mieux comprendre la circulation des récits sur l'enfant " anormal » et les influences des politiques cantonales, des lieux phares en la matière (le Burgholzhli de Zurich, Malévoz en Valais dirigé par le $\mathrm{D}^{\mathrm{r}}$ Repond, l'Institut des sciences de l'éducation de Genève notamment) et provenant d'autres pays. L'ouvrage montre bien l'émergence d'un nouveau champ, dont un congrès de psychiatrie infantile réunit les experts pour la première fois à Paris, en $1937^{1}$.

Taline Garibian met en exergue nombre d'acteurs (notamment les médecins - hommes - psychiatres), souvent insuffisamment connus voire méconnus. Cette dimension biographique est éclairante. Elle montre que leurs récits sont le plus souvent ceux d'une individualisation du mal qui désigne l'enfant - et ses parents - comme responsables (ce qui explique d'ailleurs la pratique du placement de l'enfant en institution les éloignant de la famille). L'« enfant nerveux » devient dans les années 1940 l'assise des pratiques de prévention - ou prophylaxie - de la délinquance juvénile, auquel s'adjoignent d'autres catégories comme la "dementia proecocissima", reprise au psychiatre Sancte de Sanctis (1862-1935). Cette construction sémantique nous interroge sur l'absence presque constante dans les récits des psychiatres de causes sociales, structurelles et politiques. Pour le directeur de l'Office médico-pédagogie vaudois dont relève le Bercail, c'est bien la personne humaine que la psychiatrie infantile interroge, sur trois plans : "physique, psychique et spirituel ». De même, la délinquance se doit d'être étudiée comme " problème éducatif, biologique et psychique ". Quant au directeur de l'école d'éducateur, ouverte en 1954 à Lausanne, ce sont encore les «maladies de l'âme, du cœur et de l'esprit » qu'il faut guérir. Quid des conditions de vie, de travail, de logement, d'argent et de reconnaissance sociale des milieux pauvres dont relève à l'évidence l'histoire de ces 75 ans de pédopsychiatrie? Merci à son autrice de nous donner ainsi du grain à moudre. On peut s'interroger : comment expliquer le pouvoir des psychiatres sur le champ de l'enfance? Comment comprendre l'absence de femmes médecins dans ce champ de la médecine psychiatrique infantile vaudoise? Pourquoi les médecins s'emparent-ils volontiers de l'éducation alors que les pédagogues rarement empiètent sur la médecine? Comment expliquer l'engouement de médecins psychiatres pour la psychanalyse? Comment expliquer que le développement 
des services aille de pair avec une augmentation des enfants pris en charge? Pourquoi ces derniers sont-ils issus presque exclusivement de familles pauvres voire indigentes, du moins jusqu'au milieu des années 1950 ? Pourquoi leur parole reste-t-elle si peu présente dans les archives? Les anamnèses citées dans l'ouvrage sont d'ailleurs très éclairantes pour saisir le poids des récits des médecins psychiatres, hommes.

Taline Garibian n'a pas évincé les tensions, voire les désaccords, qui existent entre les principaux acteurs et actrices autour des traitements, notamment psychanalytiques, ou sur la façon dont peut être perçu à l'extérieur un tel service (par les enseignants, par la direction de l'Office cantonal des mineurs par exemple). Elle n'escamote pas non plus les bras de fer entre services lorsqu'il s'agit de rembourser les traitements et plus généralement les rôles et prérogatives de chacun et chacune dans ce champ pluridisciplinaire, notamment entre médecins et psychologues, entre psychiatres et pédiatres, entre psychanalystes et neuropédiatres.

Il est rare qu'un auteur ou qu'une autrice s'engage sur une si longue période et perçoive si finement les transformations ainsi que la ligne de force qui donne de plus en plus de pouvoir à des médecins psychiatres " alimentés ", dans les années 1950, par les enfants que les parents leur présentent. Ceux-ci sont de plus en plus inquiets d'imaginer leur enfant " nerveusement perturbé ", " névrosé ", " atteint de psychose à évolution déficitaire " ou de "troubles somatiques et psychiques intriqués " puis d'autisme, de troubles alimentaires et d'hyperactivité, et prêts eux-mêmes à entrer dans le traitement psychothérapeutique ambulatoire (l'enfant échappant d'ailleurs de ce fait au placement en internat). Dans les années 1970 encore, deux dangers mobilisent les psychiatres : la délinquance et la prostitution. L'assise de la profession de pédopsychiatre se renforce : aux classes populaires traditionnelles, objets d'un dépistage, s'adjoignent les classes moyennes qui ont adhéré aux récits psychologisants puis médicalisants de l'époque, et qui sont elles-mêmes demandeuses de diagnostics voire de traitements.

Cet ouvrage donne à réfléchir sur un champ qui, au cours du temps, n'a fait que s'étendre entre assistance et répression, puis recours aux médicaments. Cette dernière dimension est seulement effleurée : calmants, préparations vitaminées, opothérapies... Elle formera, qui sait, un nouveau volet de l'histoire à venir, entre psychologisation et biologisation de l'enfant, au nom de sa plasticité cérébrale. 
Cetté étude est à lire pour se rappeler une fois encore que l'enfant est, pour les adultes, un objet d'investissement social, financier, symbolique. L’engagement de ces derniers, souvent avec compétence, sacrifice et foi, (me) laisse toujours l'impression que les professionnels en retirent autant, voire bien plus, que les enfants désignés et traités.

Martine Ruchat

\section{Histoire de l'éducation populaire (1815-1945). \\ Perspectives françaises et internationales \\ Laurent Besse \\ Carole Christen (dir.) \\ 2017}

\section{Villeneuve d'Ascq, Presses universitaires du Septentrion}

Cet ouvrage propose une synthèse sur un sujet plutôt méconnu, qui n’a été longtemps abordé que par les seuls militants. Dès l'introduction, les deux directeurs, Carole Christen, membre junior de l'Institut de France, connue pour ses travaux sur les Caisses d'épargne et la philanthropie au XIX ${ }^{e}$ siècle et Laurent Besse, spécialiste des maisons des jeunes et de la culture (MJC) et des équipements socioculturels au $\mathrm{XX}^{\mathrm{e}}$ (et membre du comité de rédaction de notre revue), soulignent le caractère insaisissable de l'éducation populaire et remarquent que si celle-ci a un long passé, elle n'a qu'une récente historiographie.

Le colloque qu'ils ont organisé à l'université de Lille en 2017 a permis de préciser la chronologie de cette histoire et de mettre en valeur ses lignes de force. Si aujourd'hui l'éducation populaire touche les milieux populaires en dehors de l'école, elle visait au XIX ${ }^{\mathrm{e}}$ siècle à fournir les éléments de base de l'instruction au peuple, qui en était exclu. Les auteurs font commencer cette histoire avec la fondation de la Société pour l'instruction élémentaire en 1815. Ils distinguent le temps fort de l'affaire Dreyfus, au cours de laquelle émerge l'expression "éducation populaire ", qui se concrétise dans des réalisations multiples au moment où s'impose l'école obligatoire. Le Front populaire, qui évite une expression plutôt passée du côté catholique, jette les bases de ce qui sera repris à la Libération. L'éducation populaire se centre alors sur les jeunes et hors de l'école, ce que le livre n'aborde pas puisqu'il se consacre à la longue période qui court de 1815 à 1945. 